\title{
How Novices Sketch and Prototype Hand-Fabricated Objects
}

\author{
Adrien Bousseau $^{1}$ Theophanis Tsandilas ${ }^{1,2,4}$ \\ adrien.bousseau@inria.fr theophanis.tsandilas@inria.fr \\ ${ }^{1}$ Inria \\ ${ }^{2}$ Univ Paris-Sud, CNRS (LRI) \\ Lora Oehlberg ${ }^{3,1,2}$ \\ Wendy E. Mackay ${ }^{1,2,4}$ \\ lora.oehlberg@ucalgary.ca \\ wendy.mackay@inria.fr \\ ${ }^{3}$ University of Calgary $\quad{ }^{4}$ Université Paris-Saclay
}

\begin{abstract}
We are interested in how to create digital tools to support informal sketching and prototyping of physical objects by novices. Achieving this goal first requires a deeper understanding of how non-professional designers generate, explore, and communicate design ideas with traditional tools, i.e., sketches on paper and hands-on prototyping materials. We describe a study framed around two all-day design charrettes where participants perform a complete design process: ideation sketching, concept development and presentation, fabrication planning documentation and collaborative fabrication of hand-crafted prototypes. This structure allows us to control key aspects of the design process while collecting rich data about creative tasks, including sketches on paper, physical models, and videos of collaboration discussions. Participants used a variety of drawing techniques to convey 3D concepts. They also extensively manipulated physical materials, such as paper, foam, and cardboard, both to support concept exploration and communication with design partners. Based on these observations, we propose design guidelines for CAD tools targeted at novice crafters.
\end{abstract}

\section{Author Keywords}

Sketching; physical prototyping; fabrication; collaborative design; novice designers

\section{ACM Classification Keywords}

H.5.2. Information Interfaces and Presentation: User Interfaces

\section{INTRODUCTION}

Casual product design is enjoying a renewed interest in HCI, as non-professional designers now engage in the creation and customization of a wide variety of objects [29, 26, 34]. However, most existing studies focus on professional crafters that have acquired significant expertise in their respective fields. Laymen have also received little attention in design research, where "novices" often refer to designers who are starting their career [3] and as such already have more knowledge than the audience we target. As a result, most CAD tools have been

\footnotetext{
This is the authors' version of the work. Permission to make digital or hard copies of all or part of this work for personal or classroom use is granted without fee provided that copies are not made or distributed for profit or commercial advantage and that copies bear this notice and the full citation on the first page. Copyrights for components of this work owned by others than ACM must be honored. Abstracting with credit is permitted. To copy otherwise, or republish, to post on servers or to redistribute to lists, requires prior specific permission and/or a fee. Request permissions from Permissions@acm.org.

CHI '16, May 07-12, 2016, San Jose, CA, USA

(C) 2016 ACM. ISBN 978-1-4503-3362-7/16/05 ..\$15.00

DOI: http://dx.doi.org/10.1145/2858036.2858159
}

developed based on professional design practice, such as designing objects to be produced at large volume, with high consistency, using industrial size and scale machinery. Although some enthusiastic hobbyists take the time and effort to learn professional CAD tools, most novices will find them too complex and difficult for their needs and skills.

We are interested in how to allow novices to make objects, with an emphasis on understanding the transition from sketching ideas on paper to fabricating physical prototypes. Sketching and prototyping has long been recognized as fundamental skills for professional designers. Sketching allows designers to quickly externalize and refine ideas $[15,28]$ and provides visual support to explain these ideas to clients and collaborators [8]. Similarly, rough physical prototypes allow fast and tangible testing of design ideas $[2,18]$. Our goal is to study the role of sketching and prototyping when put in the hand of non-professional users in order to inform the design of CAD software adapted to this audience.

Answering these questions requires a deeper understanding of how novices currently generate, explore and communicate their ideas, through the different phases of the design process. We framed a study around a realistic, but compressed design task in which the full process, from initial ideation to final collaborative production of a prototype, can be accomplished in a day. Since our ultimate goal is to provide tools that support informal sketching and prototyping, we focus on the use of pen-and-paper sketching and hand-crafting using junk prototyping materials (paper, cardboard, foam) [13].

This study consists of two design charrettes with six novice designers each, in which participants designed and handfabricated pairs of objects. While each participant designed its own pair of objects, our setup requires each designer to collaborate with an assistant to produce the physical prototypes. This approach allows us to observe communication strategies when participants are in turn lead designers and assistants. We describe the characteristics of the sketches according to the role of and audience for the sketch, as well as how participants interacted with others for fabrication. We conclude with a discussion of the implications for design of sketching and prototyping tools for novices.

\section{RELATED WORK}

Our research builds on previous studies on sketching, physical prototyping, collaborative design, and design practices of novice and professional designers.

Roles of Sketching. The role of sketching in creative thinking has been extensively studied in a range of domains, includ- 
ing design research, cognitive science and cognitive psychology [28]. Goldschmidt [15] observes that designers produce sketches as part of their own pictorial reasoning around a design problem, and use sketches to induce images of the entity that is being designed. Goel [14] emphasizes the ambiguity of the designer's system of internal representations during the early phases of design and argues that sketching can provide a better match with such ambiguity than structured CAD systems. Eckert et al. [7] explore the role of sketching across different design domains and identify three main roles: to generate and record ideas, to represent abstract properties pictorially, and to communicate design ideas to others. They observe that not all designers sketch to generate ideas, but for many of them the most fundamental role of sketches is to quickly communicate with others.

Sketches and Design Outcome. Other studies have explored the connection between sketching and quality of design outcomes. Song and Agogino [31] analyze the design activities of new product design teams and find that the volume of total sketches and the number of 3D sketches developed throughout the design process had a positive effect on the final design outcome. Later studies with engineering design students [38][39] do not confirm these results. Yang and Cham [39] report that sketching is not the only avenue for designers to represent their thinking. Individuals who do not draw can clarify things in their head, in prototypes, in text, or verbally through discussion.

Physical Prototypes and Materials. Designers use prototypes, or physical models of a design, to represent or explore a possible design idea. In a controlled study examining the interface between sketches and prototypes, Faas et al. [11] find that quick physical prototypes are as effective as quick sketches in order to flesh out simple design ideas. Prototypes also serve as a means to iterate on a design, immediately learn about the problem, and implement those improvements. In a study of a time-constrained prototyping task, Dow et al. [6] conclude that iterative prototyping produces higherperforming design concepts than non-iterative prototyping.

Physical materials themselves can inspire new design ideas, demonstrate properties and limitations of hardware and its components, or ground design communication among collaborators [2]. Wendrich [37] observes design students using various $\mathrm{CAD}$, graphical sketching, and physical prototyping techniques to create 3D car models from sketches. He finds that tangible interactions with physical construction material appears to add quality and detail to the end result, and enhance participants' concentration and involvement.

Collaborative Design. There are several models for how to collaborate during design [9] such as the traditional "throw the design over the wall" approach where one person passes her design to another person responsible for implementation, or a more interdependent approach where considerations from both design and implementation influence each other. Collaborative design requires constant communication through sketches and prototypes. Dow et al. [5] show that when designers share multiple prototypes with peers, they achieve better rapport in the group and better quality results.
Because design communication is rich and multimodal, an understanding of design activity must go beyond text and graphics to also include the collaboration setting and any relevant artifacts [35]. In addition to visual and physical artifacts, collaborators also communicate via hand gestures, gaze, body gestures, and visual cues [30]; the particular combination and importance of these cues will depend on the particular task. Communication modes become a major factor when supporting design teams collaborating at a distance. For example, in a distributed construction task, Kirk and Fraser [22] find that gesturing using hands is quicker than using digital sketches without any loss of accuracy. Eris et al. [10] examine the role of gesturing during design sketching. They observe that sketching and gesturing may play different roles in different phases of a compressed design process, where gesturing occurs earlier when exploring the problem while sketching emerges later when detailing the identified concepts.

Novice vs. Experienced Designers. Other work has looked at differences between novice and experienced designers. Suwa and Tversky [33] examine how professional architects and students think over their sketches and find that practicing architects could identify more functional relations in their sketches and pursue with them deeper design thoughts than students. Ahmed et al. [1] compare the behavior of beginners and expert designers from an aerospace company. They observe that beginners lack confidence in their decisions and adopt a "trial and error" strategy where they implement a solution as soon as it is found, evaluate the implementation and repeat the process if the solution is not satisfactory. Beginners also favor 3D visualization or physical manipulation of models to understand their function and assembly. Cross [3] argues that novices usually adopt a "depth-first approach to problem-solving", where they sequentially explore sub-solutions in depth, while expert strategies are predominantly "top-down and breadth-first" approaches.

\section{GOALS AND RESEARCH APPROACH}

Most of the above studies focus on professionals or student designers who are already exposed to formal training. They also examine either prototypes or sketches - there are few examples of more recent work that look at the intersection and transitions between the two. Our goal is to observe how people with no formal training in design perform collaborative design tasks, from concept to fabrication. We refer to our target audience as novices. Specifically:

- How do novices sketch for themselves (ideation) and for others (presentation and instructions)?

- How do novices fabricate prototypes with hands-on materials?

\section{- How do novices collaborate for fabrication?}

Studying design behavior is challenging because design is a complex, collaborative process that often takes place over a long period of time. Previous studies of design behavior have set up time-constrained laboratory observations of a specific, focused design task $[2,36]$, or taken an longitudinal ethnographic field study approach [19] to look at the evolution of design behavior over time. 
We combine these approaches by holding two all-day design charrettes. By defining the charrette format to have both an open-ended creative task and well-defined stages and outcomes, we can observe pairs of designers as they successively work individually and together through several phases of the design process over the course of a day. This approach lets us structure and control key aspects of the design process (e.g., design phases, materials and tools, collaboration roles, discussions) while collecting rich data from a range of sources such as sketches on paper, physical models, and videos of collaboration discussions. This structure also ensures that we can compare sketches within and across participants according to two criteria: 1 . the purpose of the sketch: ideation, development, presentation, documentation, and 2. the audience for the sketch: self, decision-maker, assistant. In the sequel, we use the term sketch to refer to representations of design concepts that may include both drawings and text.

Note that our goal is not to create a controlled hypothesistesting experiment, per se, but rather to explicitly control the instructions and the conditions for producing the sketches to maximize our ability to compare them. This study design allows us to make systematic comparisons of sketch features according to sketch purpose and audience, and to identify whether these criteria affect the design of sketching and prototyping tools for novice designers.

\section{METHOD}

The study consisted of two design charrettes. Each took place on a different day with a different group of participants.

\section{Participants}

A total of 12 volunteers, 21 to 29 years old, participated. They were divided into two groups of six participants (two women, four men), with one group participating in each charrette. The first group were all students of the same Masterslevel HCI program. The second group were again Masterslevel students, all doing an internship within the same HCI research team. Although students had taken an intensive oneweek class in HCI design methods, including brainstorming and paper prototyping, they had no training in the core skills required for the study, drawing, describing and crafting 3D objects. A self-assessment of participants on their drawing and crafting skills ("I have good skills") ranged from $[2=$ Disagree $]$ to $[4=$ Agree $]$ in a 5-item scale, where median was $[3=$ Neutral $]$. Participants were international students from seven different countries. One participant was a native English speaker.

\section{Design Brief}

To design the format of the study, we needed to work with several constraints:

Study Duration. Our design brief should be addressed within a one-day period. As a result, our task cannot require exploratory user research, detailed definition of design requirements, complex fabrication processes, or functional testing.

Skills. Given our focus on novices, we considered design tasks that are common for this audience and that do not necessitate expert skills in drawing or fabrication.

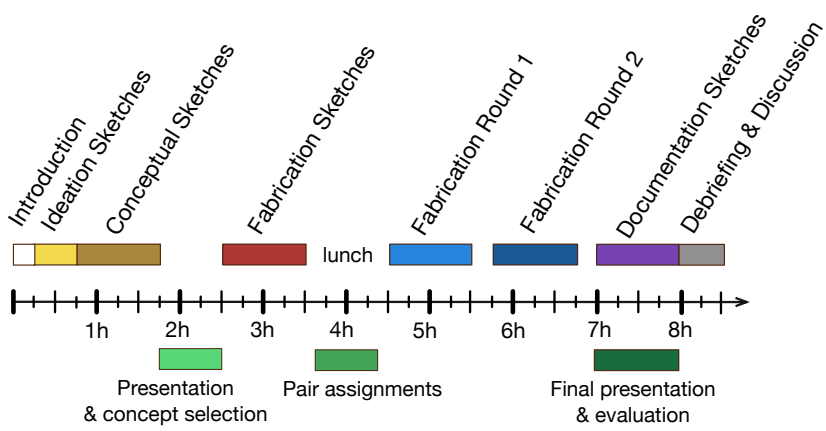

Figure 1. Overview of time allocation. The documentation phase (in purple) was only added to the second design charrette.

Materials and Tools. Advanced fabrication technology, such as $3 \mathrm{D}$ printers and laser cutters, require more training and build time than is compatible with the short duration of the study. Therefore, we focused on design tasks that could be prototyped with materials that are easy to manipulate, such as paper, foam or cardboard.

Collaborative Design. We wanted a design task that at some point would require communication and collaboration around sketches and prototypes. As a result, our task should involve multi-part concepts, where one part could easily be delegated to an assistant fabricator.

To satisfy these constraints, we framed our design brief around a costume party, where participants need to create paired costumes under a common theme. For the first charrette, the theme was Home. For the second charrette, the theme was Beach. Costume design is a common activity for everyday "makers" [34]; it quickly triggers creative and fun ideas among non-professional designers, yet does not require as much expertise as architecture, car or fashion design. Costumes can also be easily paired for couples, allowing the delegation of one of the two costumes to an assistant. Costumes can represent a wide range of physical objects that can be prototyped with a various easy-to-use materials.

\section{Task and Study Design}

We structured the design charrette as four main subtasks that emulate the common phases of professional design process [8, 18]: (1) ideation sketching, (2) conceptual sketching for presentation, (3) sketching for fabrication, and (4) fabrication. After running the first charrette, we added an additional phase to the second: at the very end we asked participants to document the fabrication of their prototypes using sketches and text such that any other participant could reproduce it. We introduced this phase in order to compare the evolution of their sketches and instructions before and after experimenting with fabrication. Figure 1 shows the exact structure and timing of the charrettes as a timeline.

Participants worked individually for all but the fourth phase (fabrication), for which they were assigned as pairs where each had a role: leader or assistant. The goal of the leader is to build one of the two paired costumes he or she designed. The goal of the assistant is to build the other costume, follow- 
ing the instructions of the leader. We randomly assigned each costume of a pair to the leader and assistant.

There were two fabrication rounds (see Figure 1). Across the two rounds, participants changed roles and worked with different people as their assistant or lead designers - this ensured that everyone had a fresh interaction with a new person with whom they did not have previous experience in a similar task. At the end, each charrette resulted in six costume pairs and a total of 12 unique costume prototypes. Six of them were created by leaders and the other six were created by their assistants.

We controlled the collaboration between the leader and the assistant by means of structured discussion sessions that interleaved with sessions of individual work time, when each participant could make progress on their prototype. Each onehour fabrication round consisted of three five-minute discussions followed by three 15-minute individual work sessions.

\section{Materials}

To help the participants prototype more quickly, we asked them to develop mock-ups of costumes on doll-sized mannequins. We provided a range of "junk" prototyping materials [13] - including blocks and sheets of foam, pipe cleaners, wire, paper, popsicle sticks, etc. - as well as small mannequins on which they could build their costume models (inset). We also provided basic tools to manipulate the materials, including cutting knives, rulers, and glue. For sketching, we provided blank A3 pages (tagged to facilitate data collection) and a variety of pens, colored markers, pencils and erasers.

\section{Procedure}

In addition to the four principal investigators, a jury of three experienced judges helped us run the study. The presence of the jury formalized the goal of the design charrette and motivated participants to best present their work. Furthermore, student volunteers assisted in documenting the charrettes by taking photographs and videos throughout the day. Below, we present the detailed procedure that we followed.

Introduction. We distributed a questionnaire to collect information about the profile of the participants. We explained the goal and overall structure of the study and presented the design brief. All instructions were provided in English.

Ideation. We asked participants to generate as many ideas of paired costumes as they could. We allowed them to use drawings and text, in whatever language they wanted. This acted as a warm-up exercise and ensured that each designer had the freedom to consider many design alternatives, resulting in greater variability of concepts among participants and triggering more diverse conversations later on.

Concept Development and Presentation. We asked participants to select three design ideas to develop and then produce presentation sketches to communicate them to an outside audience. All participants showed their sketches to the jury, who selected one "best" concept from each participant that they would continue to pursue. By letting the jury select the final concept, we avoided the possibility of participants ending up off-track simply because their concept had less opportunity for interesting development.

Fabrication Sketches. After receiving the jury's feedback, we introduced the participants to the prototyping materials, and described the afternoon's fabrication task. We then asked participants to create instructions for the fabrication of each part of their design through sketches that could include both drawings and text. We did not reveal which part would be assigned to them or to their future assistant, ensuring that they put in consistent effort to describe both parts of the concept.

Fabrication. After a quick break for lunch, we assigned participants to pairs of leader and assistant designers and instructed them about the structure of their discussion sessions. We asked them to use their fabrication sketches to support their conversations and produce additional sketches if needed.

Documentation. We only introduced this phase for the second charrette. We asked participants to create documentation sketches for the two costumes they fabricated themselves as well as the two costumes fabricated by their partners.

Final Presentation and Debriefing. At the end of the day, the final prototypes were presented to the jury and the researchers. For the second charrette, this step was run in parallel with the documentation phase. The study ended with a debriefing, when participants discussed their experience and reported on their personal strategies.

\section{DATA COLLECTION AND CODING}

Observations of design activity can be gathered and analyzed in a myriad of ways, each drawing out different types of findings [4]. Tang and Leifer [35] highlight the necessity of integrating observations of multiple aspects of design activity (conversation, drawings, and gestures) in order to more fully understand activity within a design workspace. In addition to general observations of participants' activities throughout the day, we focused our analysis on three separate data sources: sketches, discussion videos, and physical prototypes.

Sketches. The first two paper authors independently inspected all the sketches and classified them according to a detailed coding scheme (see Figure 4, as well as coding scheme in supplemental materials). The coding scheme captures the use of both textual (e.g., titles, instructions, annotations) and graphical elements of different sizes (small, medium, large) in the sketches. Furthermore, it describes the use of specific techniques to communicate (1) shape such as 3D shapes and multiple views, (2) appearance such as color and texture, and (3) fabrication process such as material, step-by-step instructions, and deconstruction. We used a 4-level scale to describe the extent to which a specific element or technique was identified: $0=$ None, $1=$ Little, $2=$ Some, $3=$ Substantial. The overall agreement score can be considered as substantial [17]: Cohen's $\kappa=.75[.73, .77]^{1}$, where we use linear weights to calculate the coefficient. The two coders discussed differences in their scores to agree on final values.

\footnotetext{
${ }^{1}$ We report $95 \%$ confidence intervals (CIs) in brackets.
} 

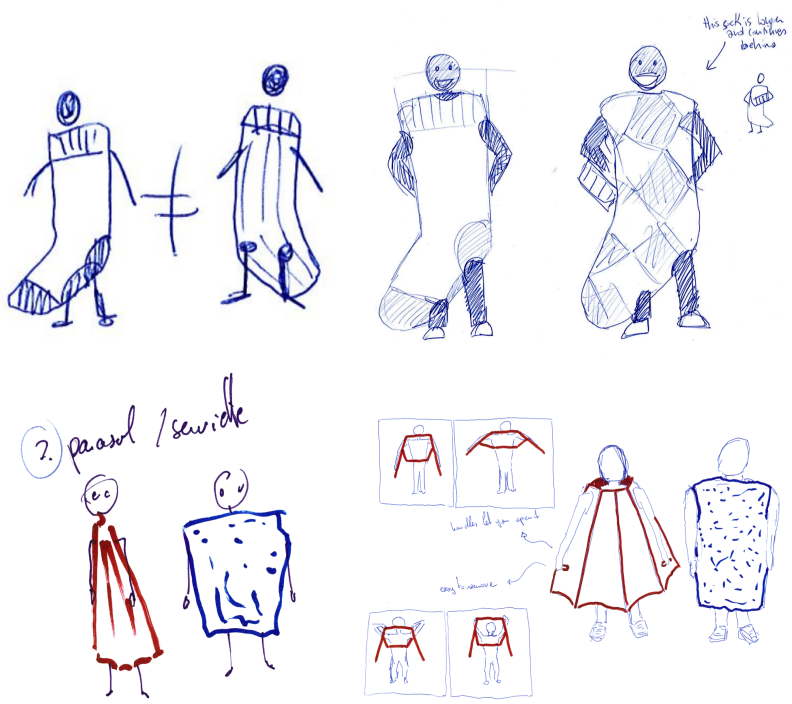

(a) Ideation sketch

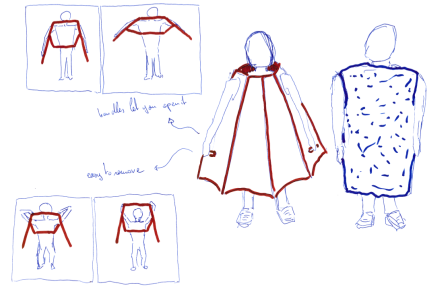

(b) Concept sketch
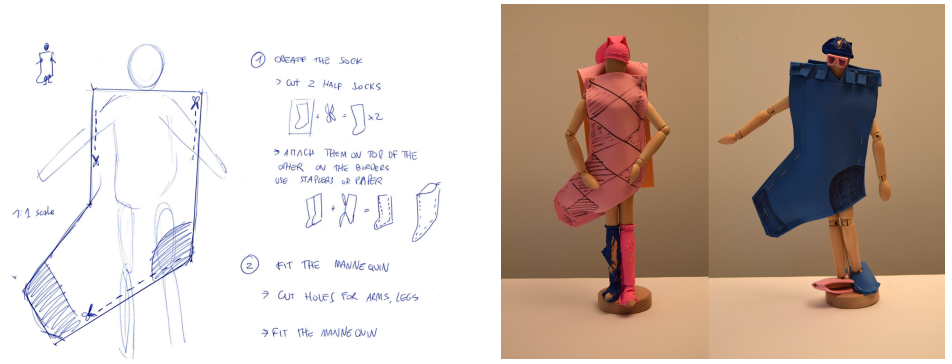

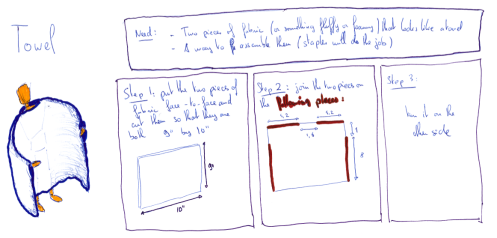

(c) Fabrication sketch

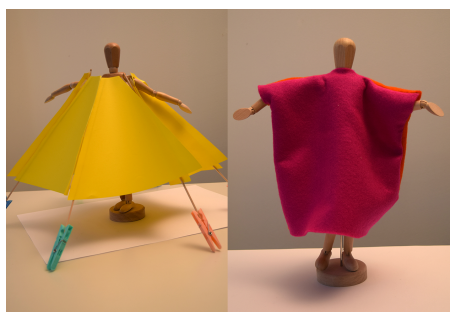

(d) Physical prototypes

Figure 2. We ran two all-day design charrettes, where participants sketched and collaborated to prototype pairs of costumes. The figure presents representative sketches and prototypes for the different phases of the study.

In addition, the two coders rated sketches across two summary dimensions (coarse vs. detailed and textual vs. graphical) by using a semantic differential scale with seven levels. For this, we use the Kendall rank correlation coefficient as a measure of agreement: $\tau=.75[.66, .82]$ (coarse vs. detailed) and $\tau=.84[.77, .90]$ (textual vs. graphical). Again, the coders discussed and agreed on final ratings.

Discussion Videos. Discussion videos were coded by the same two authors according to a coding schema and procedure on which they agreed in advance. Each coder coded a different half of the videos with ChronoViz [12]. Our coding schema captured the reference of discussion, which could be paper, the prototype (or mannequin), and air. It also captured the leader's and assistant's actions (pointing, drawing, free gestures, material use) and talking.

Physical Prototypes. We recruited 10 external evaluators to evaluate the final prototypes. All the evaluators were Master or Ph.D. students in HCI. We divided them into two equal groups, where each evaluated the prototypes of a different charrette. Evaluators inspected individually the 12 prototypes of each charrette in interaction with their fabrication sketches. They then compared (1) the quality of the fabrication instructions, (2) the overall quality of the prototypes, (3) their difficulty, and (4) how close they were to their fabrication sketches. For each criterion, evaluators used a relative 10 -item scale. We asked them to make use of the full scale ( 1 $=$ Lowest, $10=$ Highest ) whenever this was possible. However, they could use the same rating for multiple prototypes. Evaluation sessions lasted approximately 50 to 60 minutes.

We derived final ratings by taking the median of the five scores given by the evaluators of each group. Table 1 shows Kendall's W coefficients of concordance, which provide a measure of inter-rater agreement [17]. We can see that concordance level is overall higher for the similarity measure but

\begin{tabular}{l|ll} 
& Charrette 1 & Charrette 2 \\
\hline 1. Instruction Quality & $.66(<.001)$ & $.41(.02)$ \\
2. Prototype Quality & $.44(.01)$ & $.63(<.001)$ \\
3. Fabrication Difficulty & $.66(<.001)$ & $.43(.01)$ \\
4. Similarity & $.71(<.001)$ & $.72(<.001)$ \\
\hline
\end{tabular}

Table 1. Kendall's $W$ coefficients of concordance for each charrette and evaluation measure, where p-values are shown in parentheses. $W$ values closer to 1 indicate lower uncertainty and higher agreement among evaluators. The more subjective measures (quality and difficulty) have resulted in lower agreement values.

lower for others, such as quality and difficulty, where evaluation criteria are more subjective.

\section{DATA ANALYSIS}

Figure 2 presents representative examples of sketches and prototypes. Their analysis is primarily based on coding results and observations of the discussion videos.

\section{Sketches}

Previous studies of professional design $[15,28]$ and design literature [8] emphasize the different roles that sketching plays throughout the development of a concept. Rough sketches are first used to quickly externalize ideas as well as to help generate new ones. More detailed drawings are then produced to refine the concept and present it to others. Finally, precision becomes a priority for engineering drawings that document how the concept will be assembled. While the participants had no or little training in design, they adopted similar sketching strategies, as detailed next. Figure 3 presents a high-level comparison between the sketches of the first three design phases according to their level of detail (coarse vs. detailed) and content (textual vs. graphical). Friedman's non-parametric tests show that the design phase had a significant effect on both their level of detail 


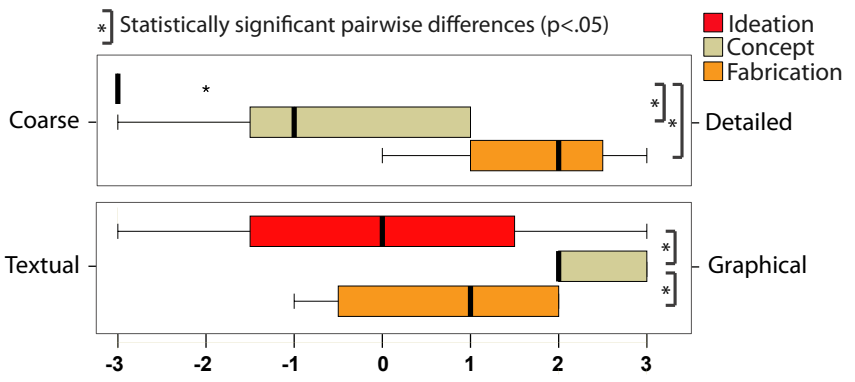

Figure 3. Boxplots summarizing the coding of participants' sketches over two dimensions: coarse vs. detailed and textual vs. graphical. We use a semantic differential scale with seven levels. Overall, participants produce more detailed sketches as they progress in the design process. We also observe that text is used for quick ideation or detailed documentation, but not for concept presentation.

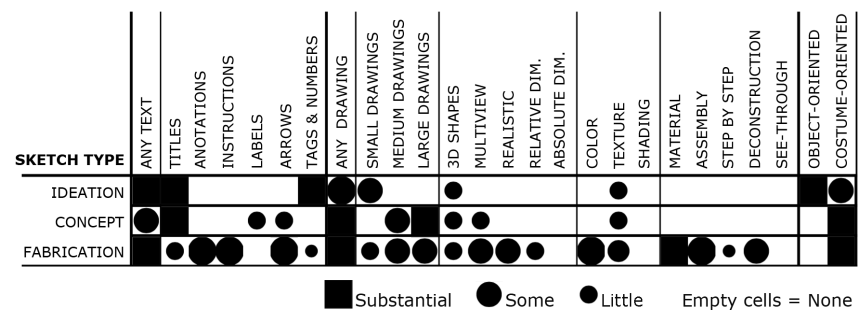

Figure 4. Tabular visualization [27] summarizing results of our coding for each type of sketch. We aggregate scores by taking median values. We observe an increasing amount of details as participants progress from small ideation sketches to larger multi-view concept sketches and, finally, more realistic fabrication sketches with additional textual and graphical elements such as annotations and assembly instructions.

$\left(\chi^{2}(2)=21.6, p<.001\right)$ and their use of textual and graphical content $\left(\chi^{2}(2)=14.0, p=.001\right)$. Figure 4 visualizes a summary of our coding results. We discuss in detail how sketches evolved across different phases below.

Ideation Sketches. During ideation, participants mainly describe their concepts with titles and small drawings. These drawings contain few details and little information about shape. Participants generated 23 ideas on average (st.dev. $=$ 16). Interestingly, the two participants who solely used text generated the greatest number of ideas ( 45 and 59 ). We believe this results from their emphasis on speed, novices who are not used to sketching can write faster than they can draw. Note that previous work [24] has shown that graphical elements result in higher-quality ideas, but we do not evaluate the quality of generated ideas here.

Concept Sketches. Participants created larger drawings for presentation, with more detail and realism than during ideation. However, sketches focus again on describing the concept, not its implementation (Figure $4,2^{\text {nd }}$ row). Concept sketches were highly graphical. Text was mostly used for titles or labels to describe context and usage of the concept. According to Sun et al. [32], text can help designers structure and better define their ideas. However, it can also hinder their quality, especially in terms of spatial relations. It seems that our participants anticipated this tradeoff and delayed the use of text for the following phase, for which the definition and communication of details were more important.
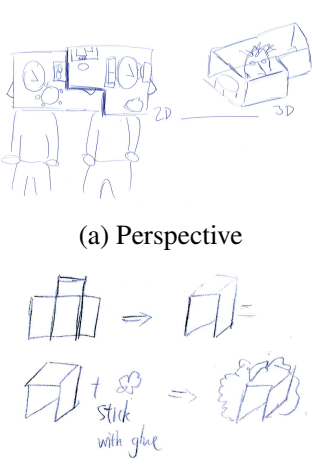

(d) Unfolding and step-by-step (a) Perspective

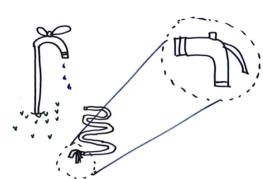

(b) Focus in context

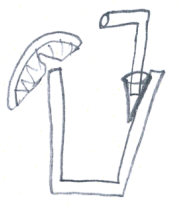

(e) Cutaway

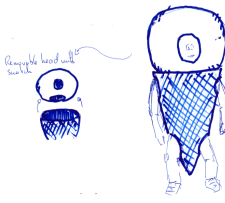

(c) Exploded view

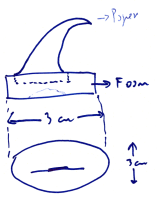

(f) Top and side view
Figure 5. Participants used a variety of drawing techniques to present their concepts (top) and plan their fabrication (bottom).

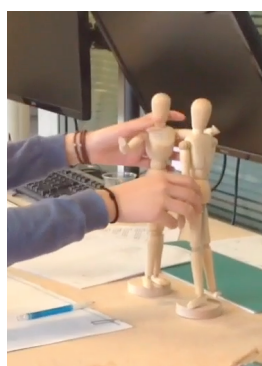

(a) Posing

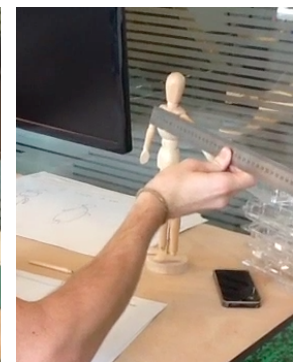

(b) Measuring

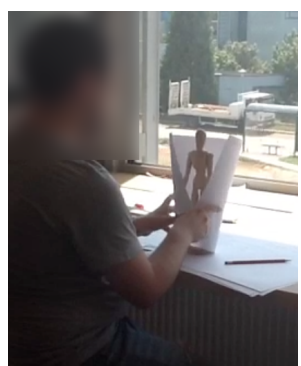

(c) Testing
Figure 6. Participants use the physical materials and mannequin to evaluate poses (a), take measurements (b), test fabrication solutions (c).

Fabrication Sketches. To describe fabrication, participants include more precise descriptions of the intended shape and its appearance. As shown in Figure 4, these descriptions are supported by textual annotations and instructions, 3D visualizations, multiple drawings to show different views of the concept, relative or absolute dimensions, as well as information on the color and materials to use, step-by-step and assembly instructions and deconstruction (unfolding, exploded views, cutaways). Figure 5 illustrates some of the drawing techniques used in concept and fabrication sketches. Overall, three main instruction strategies emerge. Some participants present the concept with a large sketch and describe its components with smaller drawings and annotations. Others use multiple medium-size sketches to present it from different viewpoints by means of perspective drawings, exploded views and unfolding of important parts. Finally, some participants combine both strategies. They present the concept with a large drawing and add textual step-by-step instructions and smaller drawings to explain how to obtain it (see Figure 2c).

Fabrication materials often guided the fabrication sketches. Several participants observed and manipulated the mannequin and materials before reporting their conclusions in the sketch. A participant declared during debriefing that "I had ideas about fabrication once I saw the material. I also got inspiration by seeing how others did." Mannequins also served as models to explore different poses or interactions between the pair of the two costumes and verify rough dimensions and proportions, as shown in Figure 6. In an observational study 


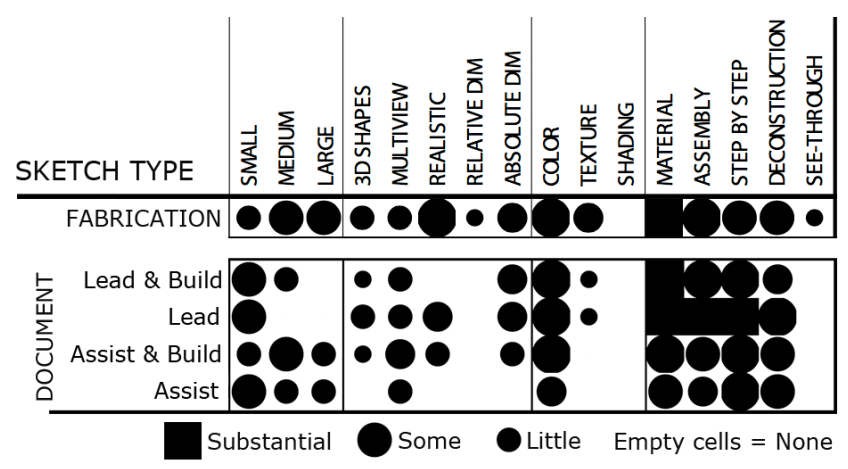

Figure 7. Documentation versus fabrication sketches for Charrette 2. We visualize [27] coding results (median values) by focusing on the graphical elements found in the sketches. Participants created documentation sketches for prototypes they built or not, as leaders or assistants. Overall, documentation sketches are similar to fabrication sketches, although participants tended to focus more on detailing fabrication steps with small drawings than on creating large realistic drawings.

on how physical objects support design thinking, Brereton and McGarry similarly noted that "Hardware and prior experiences with hardware are often the starting points from which students develop design proposals" and that manipulating hardware helps understand its properties and limits [2].

Documentation Sketches. Participants of the second charrette were also asked to produce documentation sketches after the end of the process. Each participant drew four sketches, one for the prototype they designed and fabricated (Lead \& Build), one for the prototype they designed and delegated (Lead), one for the prototype they fabricated as an assistant (Assist \& Build) and one for the prototype that the leader built while they were assistant (Assist). Figure 7 visualizes our coding of the documentation sketches, which appear very similar to fabrication sketches. However, documentation drawings tended to be smaller, especially the ones documenting costumes of participants' own designs (Lead \& Build, Lead). It seems that participants gained a more precise understanding of the fabrication process and its challenges by going through each of its steps. Having a physical prototype, several participants put more emphasis on the fabrication steps than on drawing large-scale shapes realistically. Interestingly, the realism of documentation sketches was particularly low for prototypes that did not involve a collaboration between leaders and assistants (Lead \& Build, Assist). However, these observations come from a small sample of only six participants, so their confirmation requires further future investigation.

\section{From Sketches to Prototypes}

We analyzed the ratings of evaluators to understand the transition from fabrication sketches to prototypes. Table 2 summarizes the correlations between the four evaluation dimensions.

The results suggest that better instructions (higher instruction quality) result in prototypes that are more true to fabrication sketches (higher similarity). Similarity also positively correlates with prototype quality. However, we found no clear correlation between fabrication difficulty and prototype quality. As we discuss later, participants adopted various strategies to deal with difficulty, e.g., they ended up moving away from

\begin{tabular}{l|cc} 
& Prototype Quality & Similarity \\
\hline Instruction Quality & $.31[-.05, .59]$ & $.48[.07, .77]$ \\
Fabrication Difficulty & $-.12[-.54, .36]$ & $-.45[-.80, .01]$ \\
Similarity & $.57[.18, .84]$ & \\
\hline
\end{tabular}

Table 2. Correlations (Spearman's $\rho$ ) between the rankings of the evaluators for the four measures. $95 \%$ CIs appear in brackets.

fabrication instructions to simplify implementation. The negative correlation between difficulty and similarity confirms this trend. Finally, Table 2 shows a positive correlation between instructions and prototype quality, which, however, was not statistically significant. These observations suggest that detailed sketches capture concepts that are well finalized, and as such they can be easier to reproduce as a physical prototype. In contrast, concepts described by coarse sketches need to be refined during prototyping. Faas et al. [11] made a similar observation that physical prototypes better match their sketches when the sketches include details and instructions.

We examined whether the role of the participant (Leader vs. Assistant) affected the quality of prototypes and their similarity to their fabrication sketch. The Wilcoxon signed-ranks test did not show any significant effect on prototype quality $(Z=-.52, p=.61)$ or similarity $(Z=-.72, p=.47)$. Note that leaders and assistants prototyped in parallel and interacted closely during discussion sessions. This enabled leaders to adapt their prototypes in relation to the prototype of their peers. This seems to have resulted in similar quality results and similar deviations from original designs.

\section{Discussion Sessions}

The mean discussion length ranged from $211 \mathrm{sec}(S D=125$ sec) for the $1^{\text {st }}$ session to $150 \mathrm{sec}(S D=85 \mathrm{sec})$ for the $2^{\text {nd }}$ session and $122 \mathrm{sec}(S D=67 \mathrm{sec})$ for the $3^{\text {rd }}$ session. As Eris et al. [10], we normalize time length of coded events or activities as a percentage of the total duration of a session.

We use Friedman's non-parametric test to analyze how participants' communication actions change between sessions. We found a significant effect of the session number on the use of paper $\left(\chi^{2}(2)=21.39, p<.001\right)$, the mannequin with the prototype costume $\left(\chi^{2}(2)=19.45, p<.001\right)$, and midair $\left(\chi^{2}(2)=6.53, p=.038\right)$ as reference for discussion. Figure 8 illustrates these effects, showing that the reference of discussions progressively moved from paper to the mannequin. Mid-air gestures also reduced as participants started directly referencing their prototypes during their discussion.

A further analysis showed that the quality of fabrication sketches affected the communication strategy used during discussions. More specifically, we found a positive correlation between their level of detail and the relative time spent over paper (Spearman's $\rho=.34, p=.049$ ) and a negative correlation between their level of detail and the relative time spent over the mannequin and the prototype (Spearman's $\rho=-.50, p=.002$ ), where we report partial correlations controlling for the discussion session. A possible explanation of this result is that participants tried to overcome the lack of information in fabrication sketches by spending more time discussing details over the physical models. 


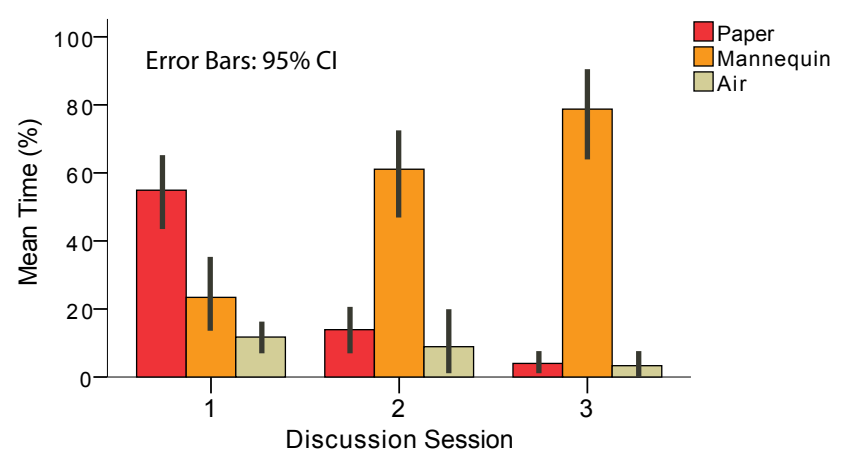

Figure 8. Use of sketches on paper, the mannequin with the fabrication model, and the air as reference for each of the three discussion sessions. We measure time as percentage of the total time of discussion.

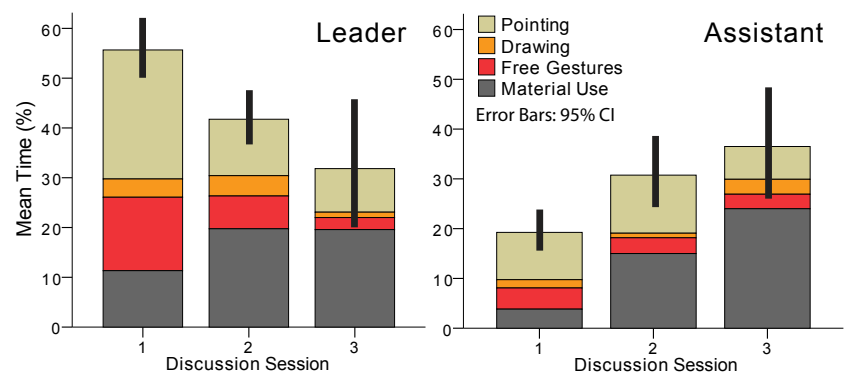

Figure 9. Use of different types of actions by the leader and the assistant. Leader actions decrease over time, while assistant actions increase. Material use progressively replaces pointing and free-hand gestures.

Figure 9 presents the proportional use of actions (pointing, drawing, free gestures and manipulation of material) by the leader and the assistant. Overall, leaders were significantly more active than assistants. Their mean activity in terms of actions was $43 \%[38 \%, 49 \%]^{2}$ as opposed to a mean of $29 \%$ $[25 \%, 33 \%]$ for assistants, which corresponds to a mean difference of $14 \%$ [ $7 \%, 22 \%]$. Similarly, leaders talked on average $50 \%[47 \%, 54 \%]$ of the time as opposed to an average of $30 \%$ [24\%,36\%] for assistants, which corresponds to a mean difference of $21 \%$ [13\%, 29\%]. The participation of the leader in terms of actions decreased with time $\left(\chi^{2}(2)=6.50\right.$, $p=.039)$, while the participation of the assistant increased $\left(\chi^{2}(2)=6.57, p=.038\right)$. As shown in Figure 9, their activity becomes balanced during the last discussion session.

We also found that pointing and free gestures had an important role during the first discussion session but were progressively replaced by the direct use of material as participants started discussing about fabrication details. Participants frequently pointed to the surface of the material, for instance to show where to cut the foam or where to attach or fasten it (Figure 10). In contrast, drawing was rarely referred to during discussions; only three participants authored new sketches to support communication. As a participant explained in the debriefing, "drawing is not enough to explain, I prefer to talk." This implies that novices prefer to interact with physical models because they lack the sketching

\footnotetext{
${ }^{2} 95 \%$ CIs have been calculated with bootstrapping methods.
}

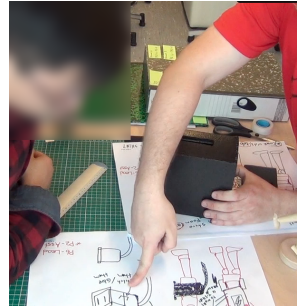

(a) Paper

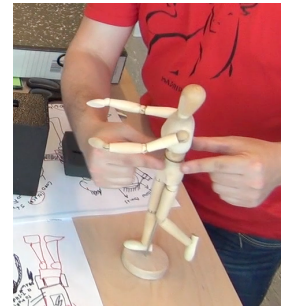

(b) Mannequin

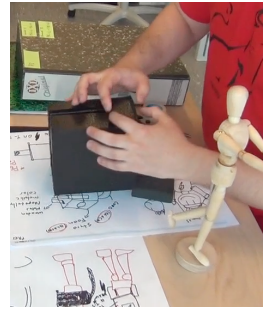

(c) Material
Figure 10. In addition to pointing to the drawing for reference (a), participants extensively manipulated the mannequin and physical materials during discussion $(b, c)$.

skills to quickly express and communicate their ideas on paper. However, discussions also reveal that novices lack intuitions about how materials behave. For example, the assistant for the toothpaste costume (Figure 11) asked about the hat: "I'm going to use a block of foam, and I need to cut into it?" The leader answered: "Yeah. Do you think that's possible?" The assistant replied with little confidence: "Let's see how my carving skills are!" The inset shows how another pair of participants manipulate a sheet of foam to discuss how it would fit on the mannequin.

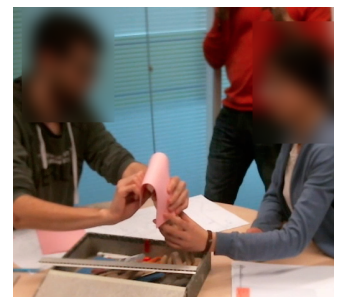

Our observations are in line with prior work on collaborative design. Tang and Leifer [35] note that pointing and gesturing allows designers to "demonstrate actions to others and focus the attention of others to specific locations," while Kirk and Fraser [22] conclude that hand gesture yield better performance than sketching. On the other hand, these results are in contrast with Eris et al. [10] who find a progressive transition from gesturing to sketching. In our case, material manipulation progressively becomes the dominant method for exploring solutions but also for detailing concepts. As Brereton and McGarry [2] observe, "hardware is used to command attention, to demonstrate and to persuade."

\section{OBSERVATIONS OF THE DESIGN PROCESS}

We selected several fabrication examples with medium to high inter-rater agreement scores. We identify several key characteristics that emerge from our observations of their design processes.

\section{Difficult Prototypes Require Good Documentation}

Most ratings suggest that similarity to the sketch is inversely correlated to difficulty. An exception is the prototypes for the "toothbrush and toothpaste" pair of costumes, which was rated as difficult, yet well-executed and similar to the original fabrication sketch. However, their fabrication sketches are also among the best-rated for quality. As shown in Figure 11, these sketches are highly detailed, providing clear instructions on which materials to use, step-by-step assembly sequences, as well as multi-view diagrams of 3D parts.

\section{Isolation Hinders Problem Solving}

The evaluators rated the "coconut" as the least similar with the lowest quality (Figure 12). This concept required build- 


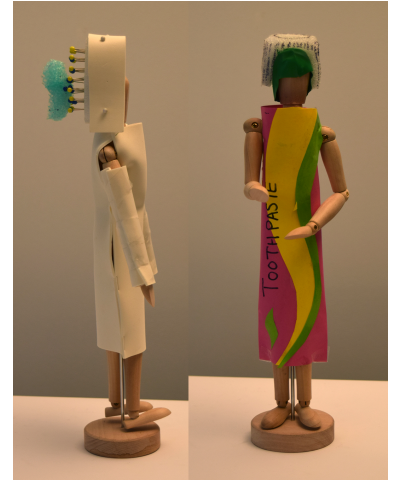

(a) Prototypes

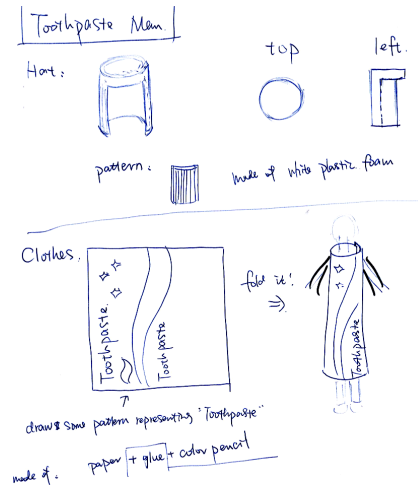

male of. paper + quet cator pencert

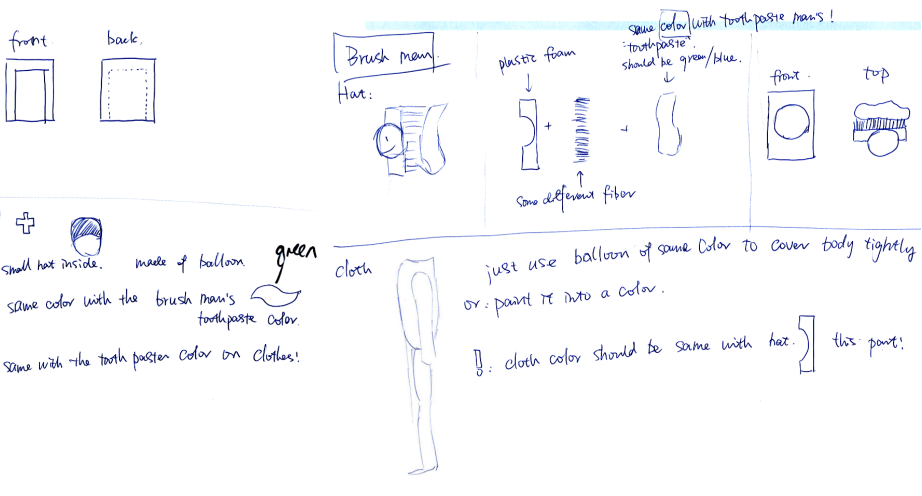

(b) Fabrication sketches

Figure 11. While judged a difficult prototype to build, the toothbrush and toothpaste prototypes (a) are highly similar to the fabrication sketches (b). These sketches were rated high in quality and include detailed instructions.

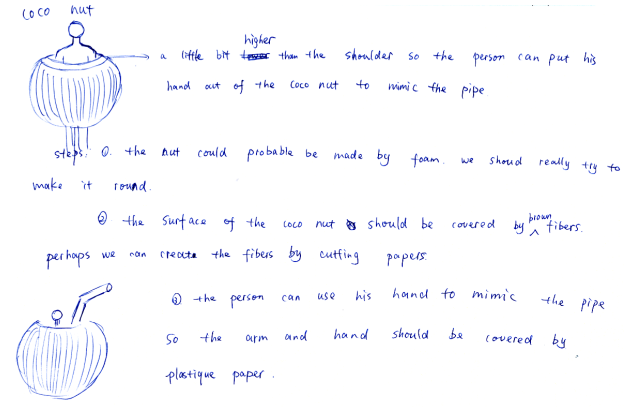

(a) Fabrication sketch

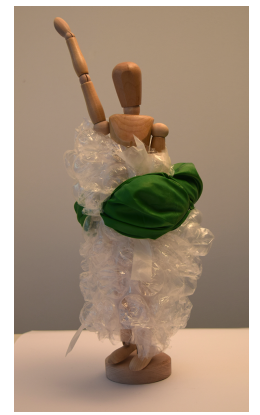

(b) Prototype
Figure 12. The leader worked on her prototype alone and did not manage to create the spherical coconut she envisioned in her sketch.

ing a sphere, which was ultimately hard to achieve with the available materials. While for the other difficult cases a solution emerged from extensive discussion between leader and assistant, the coconut was implemented solely by the leader, who did not bring it up in discussions with the assistant and as such did not benefit from any collaborative input.

\section{Pre-conceived Solutions Can Be Hard to Delegate}

Figure 13a shows the "snorkel" costume that was also rated difficult to fabricate, possibly because it involves building a curved tube. The designer anticipated this difficulty and proposed decomposing the curved part into small straight segments. Even so, the assistant had difficulty implementing this solution. Figure 13c shows snapshots of discussions, during which the leader spent significant time explaining his vision, to the point of trying the solution himself during the $3^{\text {rd }}$ session. In this pair, the leader dominated activity during the overall discussion with a $57 \%$ time participation in terms of actions compared to a $19 \%$ participation of the assistant.

\section{Unanticipated Difficulty Yields Innovative Alternatives}

Prototypes that evaluators rated as deviating most from the fabrication sketch are ones for which difficulty was not well anticipated by the designer. In particular, the "water hose" also requires the fabrication of a curved tube, but the fabrication sketch does not explain how to make a paper tube curvy (Figure 13b). During the first session, the leader ex- plained: "I thought of using paper and just folding it into the shape of a tube." The assistant replied: "If you try to do that, it will break." The assistant then proposed to cut the paper tube perpendicularly to its axis to allow bending (Figure 13d). While the assistant implemented this strategy with success, the leader obtained a result that was very different from her sketch. During the second session, the leader acknowledged: "Yes, I realized that it's very hard to make a tube!" In this group, the assistant was more active than the leader with $41 \%$ versus $29 \%$ time participation in terms of actions.

\section{MAIN FINDINGS AND IMPLICATIONS}

We discuss how this study addresses the three original research questions and suggest implications for the design of computer-aided tools to support informal sketching and prototyping of objects by novices.

\section{How Novices Sketch for Ideation and Presentation}

Despite their limited drawing and crafting skills, our participants adopted diverse drawing techniques to describe their concepts across design stages, including multiple views, perspective, unfolding and exploded views. These observations suggest that laymen are familiar with professional design imagery, even though they do not necessarily have the ability to draw them accurately. CAD tools targeting this audience must thus leverage professional drawing techniques but also offer automated guidance [20] to compensate for limited drawing skills. However, the extent to which software needs to correct for drawing errors is a subject for future studies.

\section{How Novices Fabricate Prototypes}

A key observation of our study is the critical role of physical materials in the transition from sketches to prototypes. Materials supported design exploration and testing during the sketching phase and even supplanted sketches during discussion on physical prototypes and on resolution of fabrication challenges. Participants often had difficulty anticipating fabrication challenges during the sketching phases and only resolved them through physical manipulation of materials.

This observation motivates the need for tangible interfaces to model 3D objects by manipulating physical prompts [16, 25]. However, novices lack good intuitions about how to 


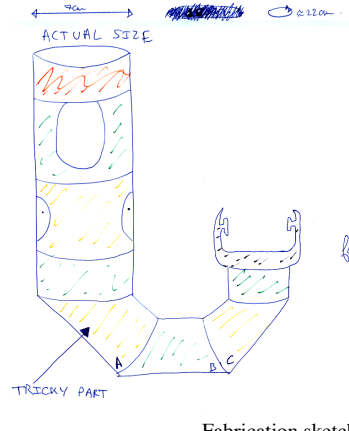

Fabrication sketch

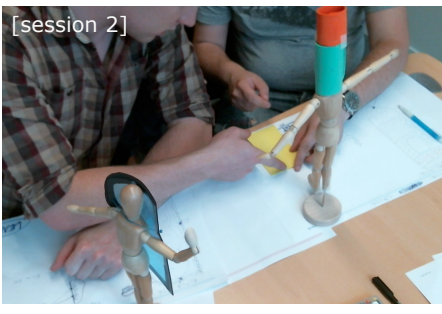

Leader: "No, it's straight line."

(a) Snorkel costume. The leader's solution was to combine small segments.

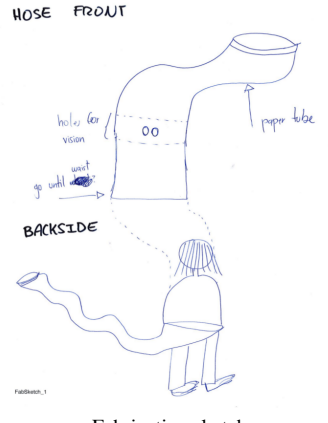

Fabrication sketch

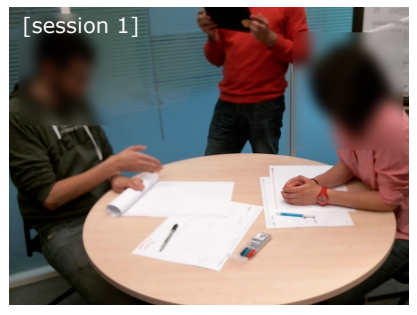

Assistant: "...and then cut it regularly."

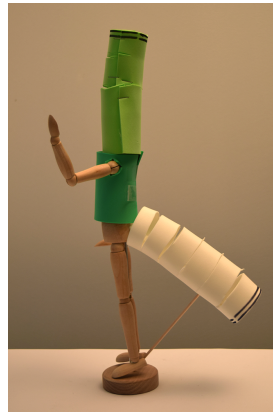

Prototype

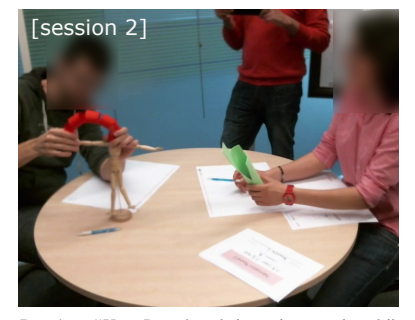

Leader: "Yes, I realized that it's very hard."

Figure 13. Both costumes present the challenge of creating a curved tube. (a) The leader understood this challenge. His sketch indicates how to build the tube from small segments. The leader spent significant time explaining this solution to the assistant. (b) The leader did not anticipate the difficulty of the fabrication task, so her sketch does not show how to curve the tube. The assistant proposed his own solution, cutting the tube to allow bending.

hand-fabricate 3D shapes from malleable materials, e.g., paper, cardboard, and foam. Therefore, CAD tools should provide instructions that take the characteristics of construction materials into account and help novices craft and assemble simple shapes such as spheres, cylinders and boxes. Such "material-aware" instructions could leverage crafting techniques used by professional designers, such as paper and cardboard folding [21]. Note that basic shapes with rough dimensions should be enough at this stage since designers are still exploring concepts rather than defining their details.

\section{How Novices Collaborate for Fabrication}

Our analysis of the discussions revealed that while the leader dominates the conversation early on, the assistant contributes more as he gains insights on the fabrication task. The focus of the discussions also progressively moved from the initial sketches to the physical prototypes. In the context of remote collaboration, this observation suggests the need for tools capable of shifting from a presentation mode where the leader explains his idea via sketches to back-and-forth critique where both participants contribute equally around a shared artifact. Since people interact differently with sketches and physical models, different collaboration stages require specialized visualization, pointing, and annotation tools.

The "water hose" and "coconut" examples suggest the benefit of collaboration to solve fabrication challenges. In both cases, the leader did not anticipate all fabrication challenges. The fabrication sketches were thus incomplete. In the "water hose", the assistant could look at the sketches, understand the concept, and propose an original solution. For the "coconut", the leader did not ask or receive any help from the assistant and ended up with a poor solution. Collaborative tools should thus encourage participants to solicit critique and feedback rather than focusing on performing the task or guiding others.

\section{CONCLUSIONS AND FUTURE WORK}

This paper describes a study of how a dozen novices with no formal design training collaborated on the design of handmade objects, using sketches, prototypes and design conversations. Each participant acted both as a lead designer and as an assistant and was involved in the design and fabrication of two pairs of objects over a one-day charrette. This design offers a unique balance between an open-ended creative design task and a well-defined study, allowing for systematic qualitative and quantitative comparisons of similar activities, within and across participants. As with every study design, it involves trade-offs. In particular, its short duration did not allow us to observe multiple design iterations.

We focused on novice designers, who have been little studied but are increasingly likely to need digital tools given the growing interest in personal fabrication. We also focused on low-cost, accessible tools and materials for sketching and prototyping. Nevertheless, we believe that our study design can be adapted for professional designers and other design tasks such as 3D modeling and printing. In particular, several sketch-based interfaces have been proposed to assist the creation of 3D models suitable for digital fabrication [23]. In this context, we would like to compare the $3 \mathrm{D}$ models produced (a) directly from sketches, or alternatively, (b) after exploring shape with a combination of sketches and junk prototypes.

\section{ACKNOWLEDGEMENTS}

This work was partly funded by ANR (ANR-12-JS02-003-01 DRAO) and the ERC grant $n^{\circ} 321135$ CREATIV. 


\section{REFERENCES}

1. Saeema Ahmed, Ken Wallace, and Lucienne T. Blessing. 2003. Understanding the differences between how novice and experienced designers approach design tasks. Research in Engineering Design 14, 1 (February 2003). DOI :

http://dx.doi.org/10.1007/s00163-002-0023-z

2. Margot Brereton and Ben McGarry. 2000. An Observational Study of How Objects Support Engineering Design Thinking and Communication: Implications for the Design of Tangible Media. In Proceedings of the SIGCHI Conference on Human Factors in Computing Systems (CHI 'O0). ACM, New York, NY, USA, 217-224. DOI : http://dx.doi.org/10.1145/332040.332434

3. Nigel Cross. 2004. Expertise in design: an overview. Design Studies 25, 5 (2004), 427 - 441. DOI : http://dx.doi.org/10.1016/j.destud.2004.06.002

4. Nigel Cross, Henri Christiaans, and Kees Dorst. 1996. Analysing design activity. Wiley.

5. Steven Dow, Julie Fortuna, Dan Schwartz, Beth Altringer, Daniel Schwartz, and Scott Klemmer. 2011. Prototyping Dynamics: Sharing Multiple Designs Improves Exploration, Group Rapport, and Results. In Proceedings of the SIGCHI Conference on Human Factors in Computing Systems (CHI '11). ACM, New York, NY, USA, 2807-2816. DOI : http://dx.doi.org/10.1145/1978942.1979359

6. Steven P. Dow, Kate Heddleston, and Scott R. Klemmer. 2009. The Efficacy of Prototyping Under Time Constraints. In Proceedings of the Seventh ACM Conference on Creativity and Cognition ( $C \& C^{\prime}$ '09). ACM, New York, NY, USA, 165-174. DOI : http://dx.doi.org/10.1145/1640233.1640260

7. Claudia Eckert, Alan Blackwell, Martin Stacey, Christopher Earl, and Luke Church. 2012. Sketching across design domains: Roles and formalities. Artificial Intelligence for Engineering Design, Analysis and Manufacturing 26 (8 2012), 245-266. Issue Special Issue 03. DOI : http://dx.doi.org/10.1017/s0890060412000133

8. Koos Eissen and Roselien Steur. 2011. Sketching: The Basics. Bis Publishers.

9. Steven D Eppinger. 1991. Model-based Approaches to Managing Concurrent Engineering. Journal of Engineering Design 2, 4 (1991), 283-290. DOI : http://dx.doi.org/10.1080/09544829108901686

10. Ozgur Eris, Nikolas Martelaro, and Petra Badke-Schaub. 2014. A comparative analysis of multimodal communication during design sketching in co-located and distributed environments. Design Studies 35, 6 (2014), 559-592. DOI : http://dx.doi.org/10.1016/j.destud.2014.04.002
11. Daniela Faas, Qifang Bao, and Maria C. Yang. 2014. Preliminary Sketching and Prototyping: Comparisons in Exploratory Design-and-Build Activities. In ASME Design Engineering Technical Conferences. DOI : http://dx.doi.org/10.1115/DETC2014-34928

12. Adam Fouse, Nadir Weibel, Edwin Hutchins, and James D. Hollan. 2011. ChronoViz: A System for Supporting Navigation of Time-coded Data. In CHI' 11 Extended Abstracts on Human Factors in Computing Systems (CHI EA '11). ACM, New York, NY, USA, 299-304. DOI : http://dx.doi.org/10.1145/1979742.1979706

13. Nancy Frishberg. 2006. Prototyping with Junk. interactions 13, 1 (Jan. 2006), 21-23. DOI : http://dx.doi.org/10.1145/1109069.1109086

14. Vinod Goel. 1995. Sketches of Thought. MIT Press.

15. Gabriela Goldschmidt. 1991. The Dialectics of Sketching. Creativity Research Journal 4, 2 (1991), 123-143. DOI : http://dx.doi.org/10.1080/10400419109534381

16. Tovi Grossman, Ravin Balakrishnan, and Karan Singh. 2003. An Interface for Creating and Manipulating Curves Using a High Degree-of-freedom Curve Input Device. In Proceedings of the SIGCHI Conference on Human Factors in Computing Systems (CHI '03). ACM, New York, NY, USA, 185-192. DOI : http://dx.doi.org/10.1145/642611.642645

17. Kilem L. Gwet. 2014. Handbook of Inter-Rater Reliability, 4th Edition: The Definitive Guide to Measuring The Extent of Agreement Among Raters. Advanced Analytics, LLC.

18. Bjarki Hallgrimsson. 2012. Prototyping and Modelmaking for Product Design. Laurence King Publishers.

19. Kathryn Henderson. 1998. On Line and on Paper: Visual Representations, Visual Culture, and Computer Graphics in Design Engineering. MIT Press, Cambridge, MA, USA.

20. Emmanuel Iarussi, Adrien Bousseau, and Theophanis Tsandilas. 2013. The Drawing Assistant: Automated Drawing Guidance and Feedback from Photographs. In ACM Symposium on User Interface Software and Technology (UIST). ACM, 183-192. DOI : http://dx.doi.org/10.1145/2501988.2501997

21. Paul Jackson. 2011. Folding techniques for designers, from sheet to form. Laurence King Publishing LTD.

22. David Kirk and Danae Stanton Fraser. 2006. Comparing Remote Gesture Technologies for Supporting Collaborative Physical Tasks. In Proceedings of the SIGCHI Conference on Human Factors in Computing Systems (CHI '06). ACM, New York, NY, USA, 1191-1200. DOI: http://dx.doi.org/10.1145/1124772.1124951 
23. James McCrae, Nobuyuki Umetani, and Karan Singh. 2014. FlatFitFab: Interactive Modeling with Planar Sections. In ACM Symposium on User Interface Software and Technology (UIST). ACM, 13-22. DOI : http://dx.doi.org/10.1145/2642918.2647388

24. Felicia L. McKoy, No Vargas-Hernández, Joshua D. Summers, and Jami J. Shah. 2001. Influence of design representation on effectiveness of idea generation. In Proceedings of the ASME Design Engineering Technical Conference, Vol. 4. 39-48.

25. Andrew Miller, Brandyn White, Emiko Charbonneau, Zach Kanzler, and Joseph J. LaViola Jr. 2012. Interactive 3D Model Acquisition and Tracking of Building Block Structures. IEEE Transactions on Visualization and Computer Graphics 18, 4 (2012), 651-659. DOI : http: //dx.doi.org/10.1109/TVCG. 2012. 48

26. Catarina Mota. 2011. The Rise of Personal Fabrication. In Proceedings of the 8th ACM Conference on Creativity and Cognition ( $\left.C \& C^{\prime} 11\right)$. ACM, New York, NY, USA, 279-288. DOI : http://dx.doi.org/10.1145/2069618.2069665

27. Charles Perin, Pierre Dragicevic, and Jean-Daniel Fekete. 2014. Revisiting Bertin matrices: New Interactions for Crafting Tabular Visualizations. IEEE Transactions on Visualization and Computer Graphics (Nov. 2014). DOI : http: //dx.doi.org/10.1109/TVCG. 2014.2346279

28. Terry Purcell and John S. Gero. 1998. Drawings and the design process: A review of protocol studies in design and other disciplines and related research in cognitive psychology. Design Studies 19, 4 (1998), 389 - 430. DOI : http://dx.doi.org/10.1016/s0142-694X (98) 00015-5

29. Daniela Rosner and Jonathan Bean. 2009. Learning from IKEA Hacking: I'm Not One to Decoupage a Tabletop and Call It a Day.. In Proc. SIGCHI Conference on Human Factors in Computing Systems (CHI). ACM, New York, NY, USA, 419-422. DOI : http://dx.doi.org/10.1145/1518701.1518768

30. Allison Sauppé and Bilge Mutlu. 2014. How Social Cues Shape Task Coordination and Communication. In Proceedings of the 17th ACM Conference on Computer Supported Cooperative Work \&\#38; Social Computing (CSCW '14). ACM, New York, NY, USA, 97-108. DOI : http://dx. doi.org/10.1145/2531602.2531610

31. Shuang Song and Alice M. Agogino. 2004. Insights on Designers Sketching Activities in New Product Design Teams. In International Conference on Design Theory and Methodology. ASME, 351-360. DOI :

http://dx.doi.org/10.1115/DETC2004-57474

32. Lingyun Sun, Wei Xiang, Chunlei Chai, Changlu Wang, and Zheng Liu. 2013. Impact of text on idea generation: an electroencephalography study. International Journal of Technology and Design Education 23, 4 (2013), 1047-1062. DOI :

http://dx.doi.org/10.1007/s10798-013-9237-9

33. Masaki Suwa and Barbara Tversky. 1997. What do architects and students perceive in their design sketches? A protocol analysis. Design Studies 18, 4 (1997), 385 403. DOI :

http://dx.doi.org/10.1016/s0142-694x (97) 00008-2 Descriptive models of design.

34. Joshua G. Tanenbaum, Amanda M. Williams, Audrey Desjardins, and Karen Tanenbaum. 2013.

Democratizing Technology: Pleasure, Utility and Expressiveness in DIY and Maker Practice. In Proc. SIGCHI Conference on Human Factors in Computing Systems (CHI). ACM, New York, NY, USA, 2603-2612. DOI : http://dx.doi.org/10.1145/2470654.2481360

35. John C. Tang and Larry J. Leifer. 1988. A Framework for Understanding the Workspace Activity of Design Teams. In Proceedings of the 1988 ACM Conference on Computer-supported Cooperative Work (CSCW'88). ACM, New York, NY, USA, 244-249. DOI : http://dx.doi.org/10.1145/62266.62285

36. Peter Törlind, Neeraj Sonalkar, Mattias Bergström, Eric Blanco, Ben Hicks, Hamish McAlpine, and others. 2009. Lessons Learned and Future Challenges for Design Observatory Research. In DS 58-2: Proceedings of ICED 09, the 17th International Conference on Engineering Design, Vol. 2, Design Theory and Research Methodology, Palo Alto, CA, USA, 24.-27.08. 2009.

37. Robert E. Wendrich. 2010. Raw shaping form finding: Tacit tangible CAD. Computer-Aided Design and Applications 7, 4 (2010), 505-531. DOI : http://dx.doi .org/10.3722/cadaps . 2010.505-531

38. Maria C. Yang. 2009. Observations on concept generation and sketching in engineering design. Research in Engineering Design 20, 1 (1 March 2009), 1-11. DOI:

http://dx.doi .org/10.1007/s00163-008-0055-0

39. Maria C. Yang and Jorge G. Cham. 2006. An Analysis of Sketching Skill and Its Role in Early Stage Engineering Design. Journal of Mechanical Design 129, 5 (2006), 476-482. DOI : http://dx.doi.org/10.1115/1.2712214 